\section{Rare Manifestation of Idiopathic Tracheobronchopathia Osteochondroplastica: Misdiagnosed and Untreated Entity?}

\author{
Abdul Rana ${ }^{1}$, Haitem Mezughi ${ }^{2}$, Shuja A. Malik ${ }^{3}$, Kanaan Mansoor ${ }^{1}$, Amro Al-Astal ${ }^{4}$ \\ 1. Internal Medicine, Marshall University Joan C. Edwards School of Medicine, Huntington, USA 2. Pulmonology, \\ Marshall University Joan C. Edwards School of Medicine, Huntington, USA 3. Internal Medicine, Nawaz Sharif Medical \\ College, University of Gujrat, Gujrat, PAK 4. Internal Medicine/Pulmonology, Marshall University Joan C. Edwards \\ School of Medicine, Huntington, USA
}

Corresponding author: Abdul Rana, ranaa@marshall.edu
Received 07/16/2020 Review began $07 / 20 / 2020$ Review ended 07/20/2020 Published 07/26/2020

๑) Copyright 2020

Rana et al. This is an open access article distributed under the terms of the Creative Commons Attribution License CC-BY 4.0., which permits unrestricted use, distribution, and reproduction in any medium, provided the original author and source are credited.

\begin{abstract}
Tracheobronchopathia osteochondroplastica (TBPO) is a rare benign disease of unknown cause, in which multiple cartilaginous or bony submucosal nodules project into the trachea and proximal bronchi. It usually occurs in men in their fifth decade and can cause airway obstruction, bleeding and chronic cough; patients are more prone to post-obstructive pneumonia and chronic lung infection in some instances. We report a case of a 69-year-old female who presented with shortness of breath and lower extremity swelling over the past couple of weeks. Echocardiography (ECHO) was consistent with heart failure with preserved ejection fraction, and she was treated with diuretics accordingly. Imaging revealed persistent pleural effusions bilaterally, more pronounced on the right side. During the course of her hospitalization, the patient coded once and had to be resuscitated. She had bronchoscopy done and pathology was consistent with TBPO. In this condition, there are numerous osseous or cartilaginous submucosal nodules in the trachea and the main bronchus and nodules are formed due to the deposition of calcium phosphate that results in the proliferation of osseous and cartilaginous structures resulting in the obstruction of large airways. Treatment for the most part is supportive and resolves around bronchodilators for symptomatic relief.
\end{abstract}

Categories: Internal Medicine, Pulmonology

Keywords: bronchoscope, bronchitis

\section{Introduction}

Tracheobronchopathia osteochondroplastica (TBPO) is an idiopathic, rare, benign pulmonary condition with an incidence of 0.01 to 4.2 per 100,000 people [1]. It was first described in 1857 and observed in an autopsy of a patient with pulmonary tuberculosis. This condition is characterized by numerous osseous or cartilaginous submucosal nodules in the trachea and main bronchus. It usually occurs in men in their fifth decade and cause airway obstruction, bleeding and chronic cough, and patients are more prone to postobstructive pneumonia and chronic lung infection in some instances. Nodules causing this condition are formed due to deposition of calcium phosphate [2]. Most patients are asymptomatic and are diagnosed incidentally. We present a case of TBPO diagnosed in a patient who presented to hospital with symptoms suggestive of acute decompensated heart failure.

\section{Case Presentation}

We present a case of 69-year-old female with past medical history significant for hypertension, hyperlipidemia and diabetes who came to the hospital with shortness of breath and lower extremity swelling that had progressively worsened over the last two weeks. These symptoms were accompanied with weight gain, orthopnea and chest pressure. She denied any chills, fevers and cough. She denied any dizziness, wheeze or changes to her vision. She also denied any history of smoking in the past and did not have any history of any underlying cardiac or pulmonary disease. At home, she was taking allopurinol $100 \mathrm{mg}$ daily for gout, glimepiride $4 \mathrm{mg}$ oral tablet twice daily, nateglinide $120 \mathrm{mg}$ and linagliptin $5 \mathrm{mg}$ for diabetes, amlodipine $5 \mathrm{mg}$ for hypertension and simvastatin $40 \mathrm{mg}$ for hyperlipidemia. At the time of admission, her labs were unremarkable for any acute abnormality except for a B-type natriuretic peptide of 2,772. Electrocardiography showed atrial fibrillation with rapid ventricular response (Figure 1) 


\section{Cureus}

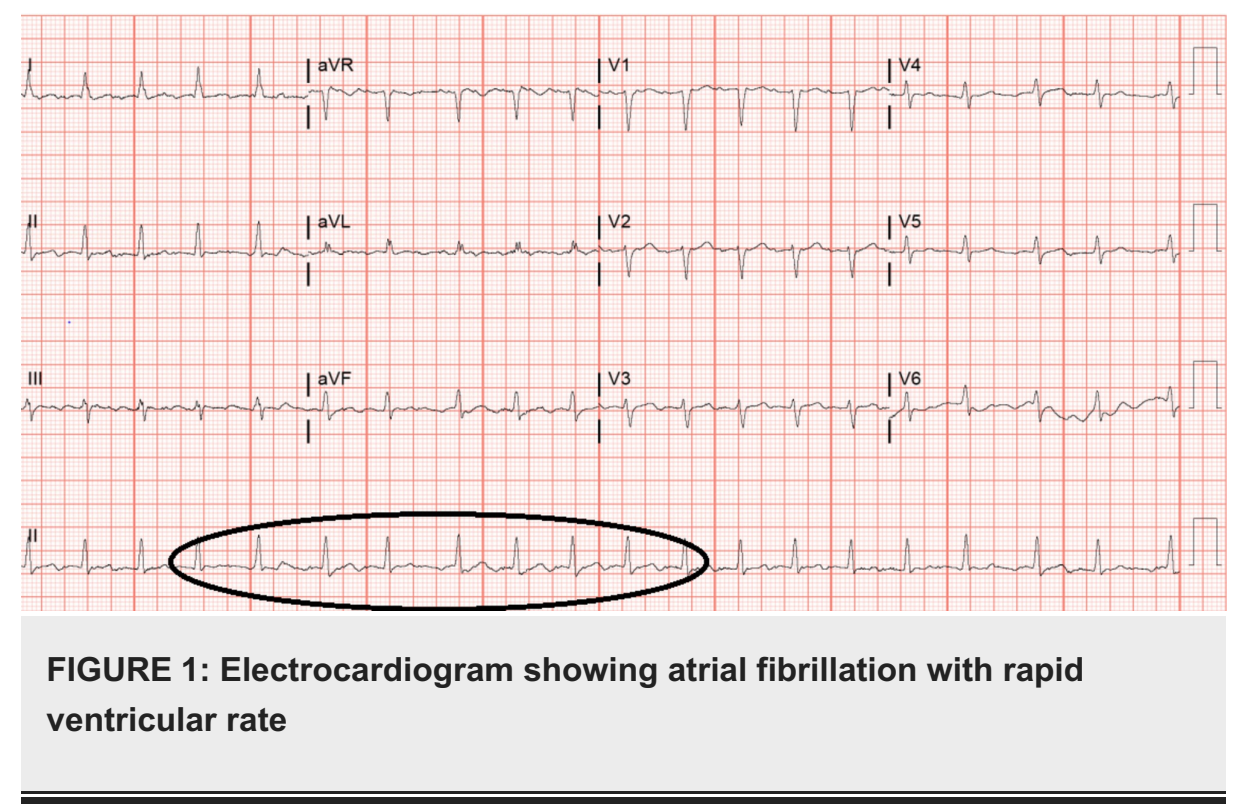

CT scan of chest without contrast showed pulmonary edema and pleural effusions right greater than left (Figure 2).

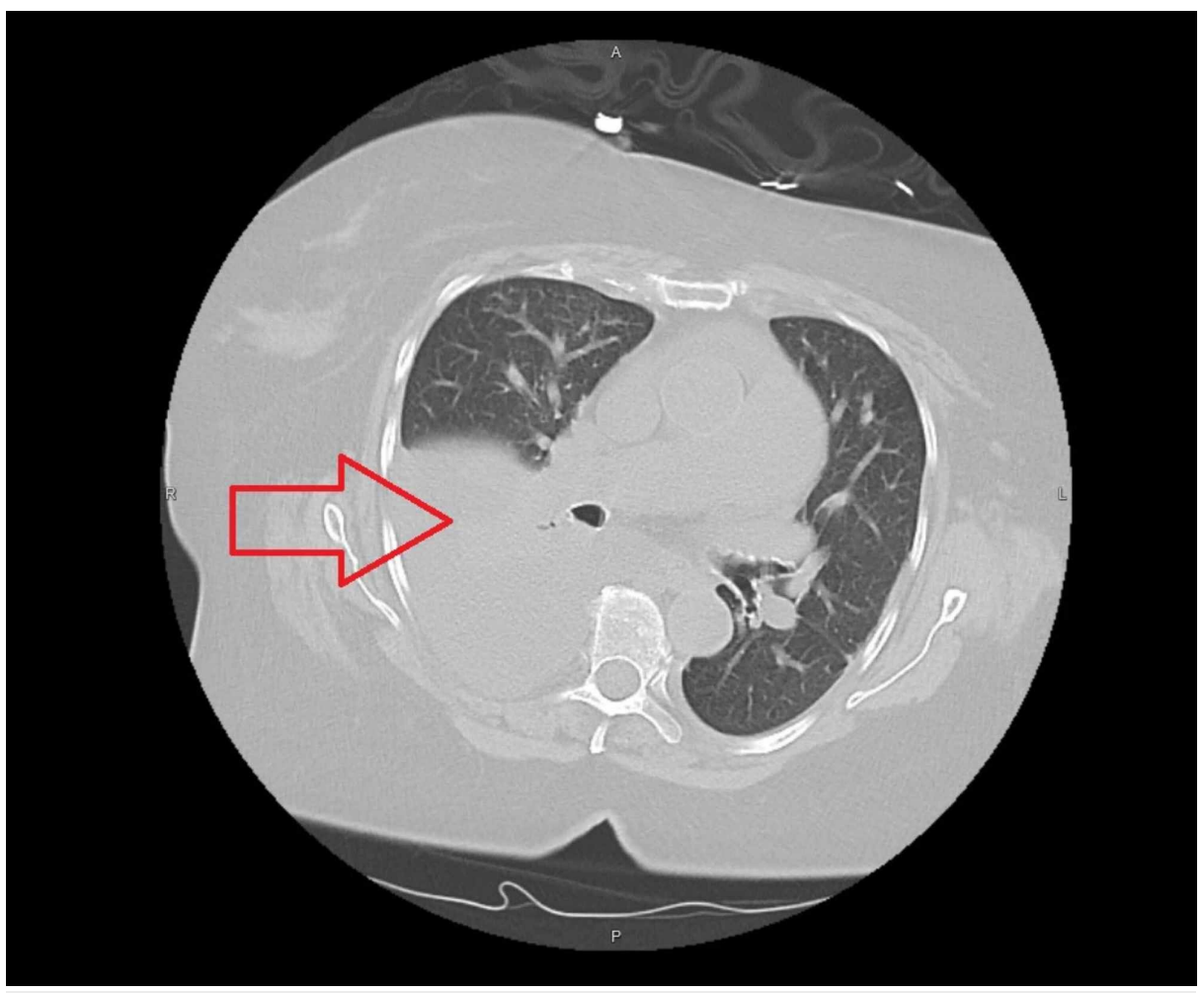

FIGURE 2: CT chest without contrast showing large right lung effusions (red arrow) with atelectasis and cardiomegaly

Cardiology was consulted and she was initially started on intravenous diuresis with $80 \mathrm{mg}$ of furosemide twice daily which was later transitioned to bumetanide drip because of persistent volume overload. Echocardiography showed findings consistent with heart failure with preserved ejection fraction $(\mathrm{EF})$. It also showed dilated left atrium with right ventricular systolic pressure of $52 \mathrm{mmHg}$ and $\mathrm{EF}>55 \%$. Pulmonology was consulted for persistent pleural effusion and thoracentesis was performed on day 4 of hospital admission. Approximately 1,100 $\mathrm{mL}$ of serous fluid was removed and fluid analysis revealed the effusion to be transudative in nature. Right heart catheterization (RHC) was performed and revealed a transpulmonary gradient of $24 \mathrm{mmHg}$ which could not be explained by only left-sided disease; hence, a Swan-Ganz catheter was left in place for hemodynamic monitoring. Other findings from RHC 
revealed wedge pressures $28 / 20 / 23 \mathrm{mmHg}$, pulmonary artery pressure of 60/36/47 $\mathrm{mmHg}$, right atrial pressure of $21 / 20 / 20 \mathrm{mmHg}$ and right ventricular pressure of 57/12/14 $\mathrm{mmHg}$; pulmonary vascular resistance of 3.3; cardiac output Fick's 9.7; cardiac index Fick's 4.06.

CT chest without contrast demonstrated large dependent pleural effusion on the right side and repeat thoracentesis was done. Approximately $1,000 \mathrm{~mL}$ of serous fluid was removed and pleural fluid analysis was unchanged from the one performed before. Post procedure film did not show any pneumothorax. The same day, the patient became bradycardic and then progressed to cardiac arrest with initial rhythm being asystole. She was resuscitated for about 8-10 minutes after which return of spontaneous circulation (ROSC) was achieved. A diagnostic and therapeutic bronchoscopy was performed. Repeat bronchoscopy was performed and revealed multiple small cysts and a large cast obstructing the right main stem consistent with plastic bronchitis that was removed. Post cardiac arrest, the patient required multiple pressor support, and repeat echo showed an EF of 20\%-25\%. Neurology was consulted because of her persistent areflexia and deemed her overall prognosis to be poor. Eventually, the family pursued comfort care measures for her and the patient passed away the following day.

\section{Discussion}

TBPO is an idiopathic, rare, benign pulmonary condition with an incidence of 0.01 to 4.2 per 100,000 people [1]. It was first described in 1857 and was observed in an autopsy of a patient with pulmonary tuberculosis [2]. In TPBO, there are numerous osseous or cartilaginous submucosal nodules in the trachea and the main bronchus as implied by the name. The nodules are formed due to the deposition of calcium phosphate that results in the proliferation of osseous and cartilaginous structures resulting in the obstruction of large airways [3]. Most patients are asymptomatic and diagnosis is incidental based on bronchoscopy or during a troublesome intubation [4]. The mean age of diagnosis is 50 years, although it has been reported in a patient as young as nine years old [5].

TBPO is definitively diagnosed by bronchoscopy during which the endobronchial nodules are biopsied and sent for histopathological analysis. Patients who experience symptoms might undergo imaging in the form of a chest CT scan prior to an invasive procedure [6]. Bronchoscopy, however, is most definitive diagnostic tool. Imaging may show obstruction in the larger pulmonary airways. The severity of symptoms depends on the location and spectrum of obstruction of the airways. These diagnostic modalities may also be supplemented by spirometry and pulmonary function tests [6].

Leske et al. studied 41 subjects with chronic cough alongside dyspnea and sputum production to be the most common symptoms. Other symptoms include chronic cough, hemoptysis, wheezing, fever, weight loss, chest pain, dysphagia and dysphonia [7]. Treatment depends on disease presentation; asymptomatic cases are not treated, whereas symptomatic ones are treated appropriately. Conservative management modalities include air humidification, avoiding irritants of the respiratory pathway and infection control. Prior studies have indicated different invasive treatment modalities, yet the results have not been promising. A case with tracheal stenosis was treated with laser therapy with only moderate improvement, whereas another case which included the excision of nodules did not result in the improvement of atelectasis post-treatment. No specific therapy exists for this condition and management is usually directed towards relieving symptoms and treating pulmonary infections when indicated [8].

\section{Conclusions}

TBPO is an idiopathic, rare, benign pulmonary condition with various presentations. Incidence is low and the disease is characterized by various manifestation. It is diagnosed with a bronchoscopy and treatment focuses on symptomatic relief. Our patient was incidentally found to have this condition where the overall clinical picture was clouded by acute decompensated heart failure complicated with asystole.

\section{Additional Information \\ Disclosures}

Human subjects: Consent was obtained by all participants in this study. Marshall University School of Medicine issued approval N/A. Deferred for case report retrospective study. We also confirm that patient consent was taken and all identifiers have been removed from study. Conflicts of interest: In compliance with the ICMJE uniform disclosure form, all authors declare the following: Payment/services info: All authors have declared that no financial support was received from any organization for the submitted work. Financial relationships: All authors have declared that they have no financial relationships at present or within the previous three years with any organizations that might have an interest in the submitted work. Other relationships: All authors have declared that there are no other relationships or activities that could appear to have influenced the submitted work.

\section{References}

1. Fois AG, Arcadu A, Santoru L, et al.: Tracheobronchopathia osteochondroplastica: a rare case report of a non-smoker and non-atopic patient, with a long history of wheezing since childhood. Multidiscip Respir 


\section{Cureus}

Med. 2016, 11:16. 10.1186/s40248-016-0050-7

2. Riva G, Girolami I, Luchini C, et al.: Tracheobronchopathia osteochondroplastica: a case report illustrating the importance of multilevel workup clinical, endoscopic and histological assessment in diagnosis of an uncommon disease. Am J Case Rep. 2019, 20:74-77. 10.12659/AJCR.911859

3. Ulasli SS, Kupeli E: Tracheobronchopathia osteochondroplastica: a review of the literature . Clin Respir J. 2015, 4:386-391. 10.1111/crj.12166

4. Tadjeddein A, Khorgami Z, Akhlaghi H: Tracheobronchopathia osteoplastica: cause of difficult tracheal intubation. Ann Thorac Surg. 2006, 81:1480-1482. 10.1016/j.athoracsur.2005.04.013

5. Simsek PO, Ozcelik U, Demirkazik F, Unal OF, Orhan D, Aslan AT, Dogru D: Tracheobronchopathia osteochondroplastica in a 9-year-old girl. Pediatr Pulmonol. 2006, 41:95-97. 10.1002/ppul.20311

6. Ribeiro GM, Natal MR, Silva EF, Freitas SC, Moraes WC, Maciel FC: Tracheobronchopathia osteochondroplastica: computed tomography, bronchoscopy and histopathological findings. Radiol Bras. 2016, 49:56-57. 10.1590/0100-3984.2014.0056

7. Leske V, Lazor R, Coetmeur D, Crestani B, Chatté G, Cordier JF, Groupe d'Etudes et de Recherche sur les Maladies "Orphelines" Pulmonaires (GERM"O"P): Tracheobronchopathia osteochondroplastica: a study of 41 patients. Medicine. 2001, 80:378-390. 10.1097/00005792-200111000-00004

8. Wang N, Long F, Jiang S: Tracheobronchopathia osteochondroplastica: two cases reports and review of literature. Medicine. 2016, 95:3396. 10.1097/MD.0000000000003396 